Review

\title{
A Review on the Role of Color and Light in Affective Computing
}

\author{
Marina V. Sokolova ${ }^{1,2}$ and Antonio Fernández-Caballero ${ }^{2, *}$ \\ ${ }^{1}$ Department of Safety of Vital Activity and Environment, South-West State University, \\ 50 let Oktyabrya, 94, 305040-Kursk, Russia; E-Mail: marina.v.sokolova@ gmail.com \\ ${ }^{2}$ Instituto de Investigación en Informática de Albacete, Universidad de Castilla-La Mancha, \\ 02071-Albacete, Spain \\ * Author to whom correspondence should be addressed; E-Mail: Antonio.Fdez@uclm.es; \\ Tel.: +34-967599200; Fax: +34-967599224.
}

Academic Editor: Takayoshi Kobayashi

Received: 13 May 2015 / Accepted: 30 July 2015 / Published: 5 August 2015

\begin{abstract}
Light and color are ubiquitous environmental factors which have an influence on the human being. Hence, light and color issues have to be considered especially significant in human-computer interaction (HCI) and fundamental in affective computing. Affective computing is an interdisciplinary research field which aims to integrate issues dealing with emotions and computers. As a consequence, it seems important to provide an updated review on the significance of light and color in affective computing. With this purpose, the relationship between $\mathrm{HCI} /$ affective computing and the emotions affected by light and color are introduced in first place. So far, there is a considerable number of studies and experiments that offer empirical results on the topic. In addition, the color models generally used in affective computing are briefly described. The review on the usage of color and light in affective computing includes a detailed study of the characteristics of methods and the most recent research trends. The paper is complemented with the study of the importance of light and color from demographic, gender and cultural perspectives.
\end{abstract}

Keywords: color; light; affective computing; human-computer interaction; review 


\section{Introduction}

Affective computing is an essential part of current human-computer interaction (HCI). This area of research provides important issues which include automated human affective behavior recognition. This type of recognition is provided by means of the capture and analysis of implicit information about the user, such as vocal and facial changes, alterations of body movements and gestures, and so on. Recognition of affective behavior is a part of affective interaction where a computer approaches the emotion, mood, attitude and personal preferences of the person [1,2]. Adding the ability of emotional intelligence to computers makes them more coherent to the humans who interact with them. Light and color are omnipresent environmental factors which influence the human being. Thereby they are key factors for affective interaction.

This paper introduces and surveys some recent advances described in the research literature dedicated to the nature of the relationship between humans and both light and color factors. It examines the state of the art in the area of the study of light and color. Information about light and color perception and preference is taken from different fields, which include affective computing, design, human-computer interaction, psychology, and medicine, among others. We review and analyze new findings and draw conclusions regarding the relation of color and light with emotion.

This paper is organized as follows. Section 2 introduces affective computing and its relation with emotions, and reviews the emotional states that are affected by light and color. Section 3 describes the most outstanding color models used in affective computing. Section 4 provides a detailed review of light and color management in the area of HCI. Then, the importance of light and color as affective factors including demographic, gender, and cultural issues is discussed. Later, an overview of the characteristics of the methods and recent research studies is introduced. Section 5 concludes with a summary and a discussion of some of the challenges that researchers are addressing in this field.

\section{Affective Computing and Emotions}

\subsection{Affective Computers in Relation to Emotions}

The notion that emotional states affect human rational thinking is at the core of affective computing. Affective computing is an interdisciplinary research field which makes efforts to integrate issues dealing with emotions and computers [3]. According to Picard, affective computing "relates to, arises from, or deliberately influences emotions". Emotions provide the power to regulate and control unconsciously many aspects of everyday life [4]. That is why the intelligent management and regulation of emotions is beneficial for everybody, in such environments as home and workplace.

Emotions include complex patterns of changes caused by feelings, cognitive processes and behavioral reactions. They are triggered in response to perceived stimuli and are personally significant [5]. Various theories and models on how the brain experiences an emotional stimulus have been proposed $[4,6]$. As noted by Brave and Nass [7], there is consensus among the theorists of emotion up to now. There exists two contrary opinions on the nature of emotion. Evolutionary theorists believe that emotions are innate as an environmentally conditioned heritage from our ancestors (see [8-10]), while, on the other hand, many experts in affections assert that emotion is mainly a social phenomenon $[11,12]$. And there are defenders 
of "basic emotions". These authors argue that there exist universal emotions shared by all humans, independently of their cultural backgrounds [13]. Damasio [14] suggests two different mechanisms that produce emotions, which are called primary and secondary emotions. A primary emotion is automatic and is generated as a momentary reflective reaction to a stimulus. A secondary emotion is more logical, and the brain interprets and sends signals to produce it. Picard [3] presents a computerized mechanism based on Damasio's model of primary and secondary emotions, suggesting that emotional states are accessible from both the inference and decision making levels.

Many scientific findings confirm that humans treat computers (and mass media in general) in the same way that they behave towards human colleagues, being polite or feeling angry or irritated [15]. Thus, a computer response in an appropriate tone, corresponding to a person's emotional state, has more possibilities of being positively accepted than a mere technical response. The principal ambition of affective computing consists in trying to "humanize" computers by making them emotionally coherent to the humans who interact with them. Indeed, Picard notes that the primary goals of affective computing are to enable computers to recognize, express, "have" emotion, and finally, be able to demonstrate emotional intelligence $[3,16]$.

In most affective HCI approaches, the final goal is either to measure user affect for the sake of doing something useful with it or to influence the user's emotion $[17,18]$. The idea of having this closed loop in which human affect plays a key role in the interaction between the human and its technology has been called the affective loop. Affect refers to feeling, emotion, mood, attitude, preferences and personality traits. The affective loop usually consists of a five step process involving different affective abilities of the technology [3]: user affect detection, user affect interpretation, system affective state synthesis, synthetic affective state expression, and user affect influence. So, affective computing deals with emotions, knowing that they have an influence upon our memory, learning abilities, perception, decision making, prioritizing, attention and motivation, among others [19]. For instance, a user might be involved in a constant affective interaction during which wearable computers collect information about his/her emotional state (and then update it regularly) and provide it for affect recognition.

Another important feature of an affective computer is emotional intelligence. As mentioned by Salovey and Mayer [20], emotional intelligence is "a type of social intelligence that involves the ability to monitor one's own and others' emotions, to discriminate among them, and to use the information to guide one's thinking and actions". Emotional intelligence is an influencing element of human-computer interactions, and its role in human intelligence and interactions is very important. This is why many researchers have dedicated their effort to study the theoretical basis of emotional intelligence and have developed practical applications [20-23]. Mayer and Geher [21] and Salovey and Mayer [20] introduce theoretic explanations and general materials on emotional intelligence, while Picard et al. [23] reports about a prototype of machine-based emotion recognition. Modeling emotions through integration of emotional intelligence into beliefs of a virtual agent is also presented [22].

\subsection{Emotions Studied in Affective Computing}

Besides the typical popular beliefs which tend to construct some unsophisticated ideas as "red is stimulating" and "blue is tranquil", as truly mentioned by O'Connor [24], there is a considerable number 
of studies which presents empirical results on the topic. Indeed, color is strongly related to emotional states as it will be shown later on. One of the most interesting experiments that proves the hypothesis about the importance of color as a determinant factor in transmitting emotions is [25]. The study consists in carrying out various experiments which show drawings made by psychologically healthy and troubled people, both in chromatic and achromatic condition. The aim is to evaluate the influence of color on the number of right guesses of the participants. Each participant has to indicate which of the two presented pictures has been drawn by a psychologically healthy or unhealthy person. Surprisingly, the number of correct guesses in the case of chromatic drawings is noticeably higher than that of achromatic ones. Another interesting finding is that healthy people use more colors for their drawings than their counterparts. Indeed, psychologically problematic people use a very limited color palette including red, black, and sometimes a few other colors.

In addition, emotional changes in people who stay in colored environments is shown. For example, affect change in painted rooms with different orientations to the window has been investigated [26]. The experiment is aimed at discovering potential relations between emotional changes and the following color groups: warm/cool, strong/light chroma, yellow/pinkish/greenish/bluish. Emotional changes in participants are evaluated through the eight primary Plutchik's emotions [27]. The author reaches some interesting outcomes, which allow relating color to emotions. In another research, it is shown that staying in rooms painted in bubble-gum pink (Baker-Miller pink) produces a sedation effect [28]. It is also outlined that muscle strength reduces within 2.7 seconds for prison inmates who stay during limited time periods in specially pink painted holding cells.

In a study of color-emotion associations by Kaya and Epps [29] it is revealed that principal hues comprise the highest number of positive emotions. They are followed by intermediate hues and achromatic colors, which induce the decline of positive emotional response. Based on the obtained responses, the green color attains the highest number of positive emotions, followed by yellow, blue, red and purple. Among the intermediate hues the results of the most preferred include blue-green, red-purple, yellow-red and purple-blue. For achromatic colors the most attractive one is white, followed by black and, finally, gray. Another experiment seeking to evaluate the effects of color images on mental stress reduction is [30]. The participants are shown colored photographs of natural scenery. Each image mainly comprises one of five key colors: pink, green, blue, yellow and red. The same series of images, now in black and white, are prepared for the control group. The color images are presented in the following order: pink, green, blue, yellow, and red. After that, biochemical and emotional parameters (salivary chromogranin-A and salivary cortisol) are measured. A multiple mood scale test demonstrates decrease in depression and fatigue and growth of positive factors such as well-being and intimacy.

In addition,, Lee et al. [31] report about a set of experiments carried out to relate emotional states with colors. The authors put forward the following three hypotheses: emotion-judgment congruence; emotion-target congruence; and emotion-target incongruence. The first hypothesis postulates that people use their current affective state to evaluate a given object. So, people in a happy/sad mood would be inclined to a more positive/negative evaluation of color stimuli (independently of the color tone) than they would if they were in an emotionally neutral state. The second hypothesis, namely "emotion-target congruence", suggests that the congruence between the emotional state and emotional tone of the target is the dominant of the evaluative judgments. "Happy colors" are preferred by happy people and "sad 
colors" are preferred by sad people. The third hypothesis, "emotion-target incongruence" states, after the findings, that people spontaneously get or maintain a positive affective state, which means that negative affect may increase preference for the stimuli that make people feel better, and positive affect may decrease preference for stimuli that make people feel worse. Consequently, sad people would approach an emotionally incongruent tone, while happy people would avoid it. The results demonstrate that the first and the third hypotheses are inconsistent and confirm the viability of the "emotion-target congruence" hypothesis (especially for sad people) with the following particularities:

- Happy people increase their preference for happy colors, and sad people increase preference for sad colors. This preference toward emotion-target congruence appears regardless of one's initial preference for the colors.

- When a colored object conveys one's attitudinal commitment, emotion-target congruence is likely to operate. However, when preference for a colored object is based mostly on utilitarian reasons and hence provides little signals about the individuals' attitudes, values and beliefs, the emotion-target congruence effect disappears.

- In case a negative emotion contains a committed attitude and meaning toward a situation (e.g., loss of loved one, break-up, or exposure to some else's suffering), the emotion-target congruence effect is likely to emerge. Preference for emotion-target congruence tends to be observed for people experiencing negative emotions of loneliness, sadness or regret.

- When a negative emotion has less committed values and meaning, emotion-target incongruence effects are more likely to emerge. In this case, preference for emotion-incongruent aesthetic stimuli tends to be observed when the emotion is induced by a not-committed negative emotion.

- Participants who are exposed to sad video and music feel significantly worse than those exposed to happy video and music.

- Participants' emotional state does not impact their reporting of color tone on the color categorization task; nor does it influence their categorization speed.

In another experiment, the influence of light and color on psychological mood has been investigated, and the outcomes have demonstrated that emotions are stronger in colored environments [32].

\section{Color Models Used in Affective Computing}

There are many definitions of the term "color". The Oxford Dictionary gives us the following one: "The property possessed by an object of producing different sensations on the eye as a result of the way it reflects or emits light". Another definition says: "One, or any mixture, of the constituents into which light can be separated in a spectrum or rainbow, sometimes including (loosely) black and white". The description of color can be modified or specified depending on the application or particular reference. Indeed, color is one of the essential components of the perception of the world, and it is one of the fundamental factors of interest for a number of research areas, as it is shown in Figure 1. 


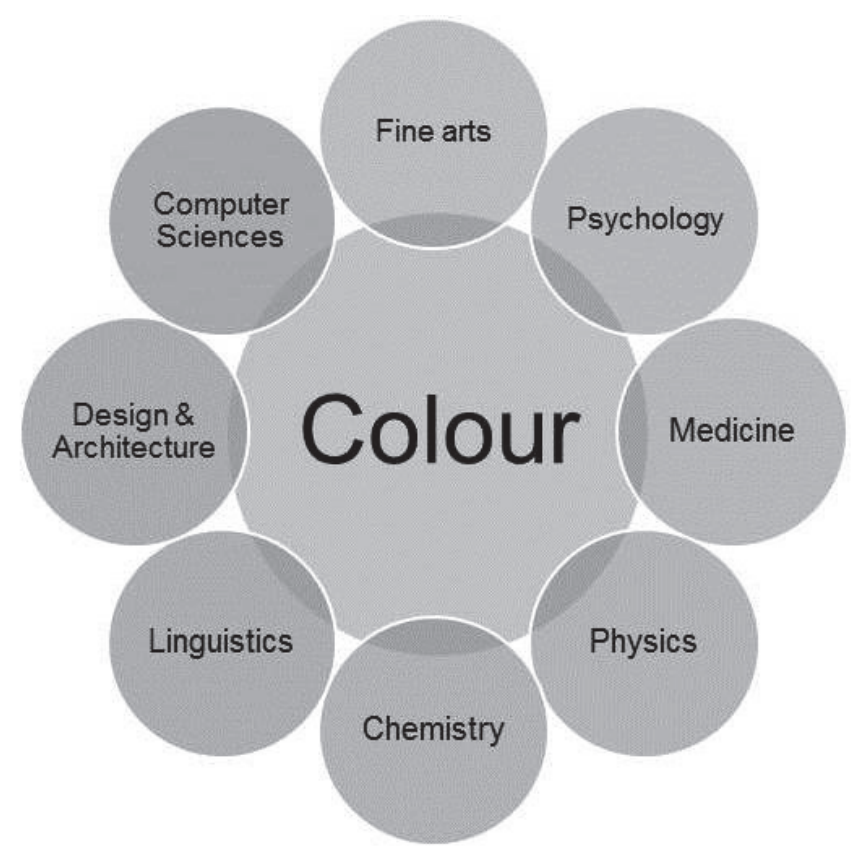

Figure 1. Color as a topic of interest to many research fields.

Various attempts to study color have later converted into classification systems. These systems facilitate understanding the complex nature of color. One of the earliest systems is named "The International Commission on Illumination" (or CIE from its French title, Commission Internationale de l'Éclairage) [33] since 1931. Each color is defined with a three primary color basis. It means that each color is measured and described by a set of values (XYZ) which indicate amounts of red, green and blue light. Later, CIE introduced CIELAB and CIELUV, modifications of the CIE system, which suit best for colorant and lighting industries, respectively. Yet, one of the shortages of the CIE system remains to be its inability to define tolerances based on the hue component.

The Munsell color system solves this problem as each color is classified by hue, value, and chroma [34]. Figure 2 illustrates the CIE and Munsell color models. The latter is shown as an orb around whose equator a band of colors runs [35]. Two other variations of the Munsell's system, namely the HSB/HLS models also use three axes to define a color. In the case of HSB, the axes are hue, saturation and brightness; and for the HLS model they are defined by hue, lightness and saturation.
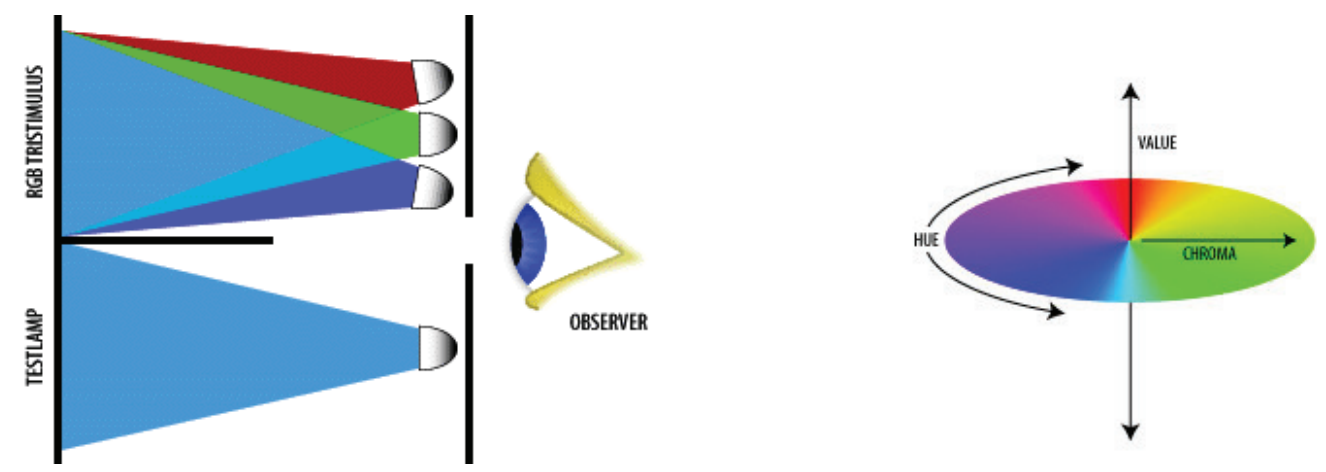

Figure 2. CIE and Munsell color models. Left: CIE color model. Right: Munsell color model. 
The natural color system (NCS) defines a color model which is based on the color opponent concept. It has been published by the Scandinavian Color Institute [36]. Each color is defined by darkness defining the amount of blackness, chroma standing for saturation, and hue determining a percentage value between two of colors red, yellow, green and blue. Lastly, the RGB additive system forms a color by using three components: red, green, and blue. NCS and RGB systems are shown in Figure 3.
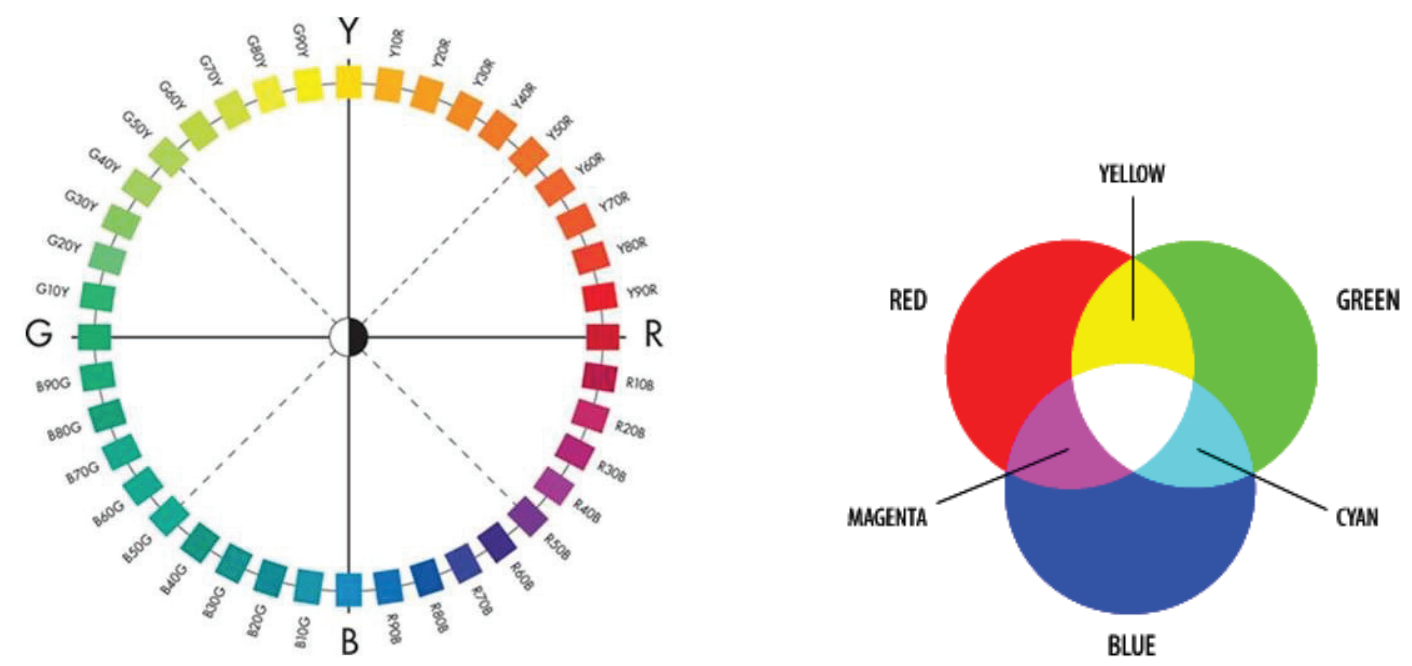

Figure 3. NCS and RGB color models. Left: NCS color model. Right: RGB color model.

The vast number of studies about the relation between color and emotion apply computer-based tests, although others use paper-based questionnaires. One of the features described in Table 1 is the color model used. They mostly include the RGB, CIE, and Munsell color systems. Moreover, there are some other models such as SCOTDIC PLUS 2000 , which is based on Munsell's color system and adjusted to textile samples [37,38].

\section{Color and Light in Affective Computing}

\subsection{Human-Computer Interaction and Color}

Color, together with other essential visual components such as shape, size, and some others, is a key component in computer interfaces. It plays an important role as an essential component of affective communication between users and computers. HCI is a totally cross-disciplinary field of research in computer science. It was introduced with the idea of enhancing software efficiency, safety and interactivity to make it closer to humans. This way, the goals of HCI meet the affective computing ones as it will be explained later on.

Beale and Peter [39] define more exactly the role of affect and emotion in HCI. They emphasize that computers become more flexible and intelligent in their interaction with users when emotions are added to them. Cyr et al. [40] have undertaken an experiment with the participation of volunteers from Canada, Germany and Japan, being them males and females. It was revealed that the website color setup is a significant determinant for trust and satisfaction. Bonnardel et al. [41] present some findings that prove the importance of color in HCI by analyzing website users' and designers' color preferences. The 
two studies carried out reveal that gray, blue and orange are preferred to green and magenta. All these findings confirm that color in HCI gains a new significance as a challenge for interaction designers.

Currently, the interaction between user and computer is explicit, though it may be performed through voice, gesture and so on, and not only by a graphical user interface [42]. The perspective is to increase traditional explicit HCI with additional abilities to attain an implicit interaction. Computers should be able to perceive and interpret instructions of a user so to include his/her emotions [43]. In other words, implicit HCI implies a certain level of emotional adaptation and recognition leading to emotional intelligence.

One central concept of HCI is usability, which means easy use and usefulness. So, attention paid to the affective component in relation to HCI should be welcome. In this sense, emotional experience has become an important topic in human-technology interaction research and design. For example, in Jokinen [44] a conceptualization of the emotional user experience based on the appraisal theory of emotion is formulated, where the goal congruence of the interaction events and the task-independent individual traits are thought to underly the user's emotional response. Additionally, color emotion patterns and schemes for design and environment organization are important in this respect.

Moreover, Lim et al. [45] evaluate emotional responses to determine the product qualities of an interactive system, offering the participants to choose between correspondent semantic values. The authors find out that the visual aspect of the product, which includes colors, material feelings, shapes, sound qualities, etc., appear to be the third most important aspect of a product, after interaction and function capabilities. In an experiment, color is one of the components which is changed by a user in order to obtain a desirable, and most mood-corresponding, view of the website interface [46]. The authors insist that the overall style and design may have a dramatic effect on the user. The proper approach towards the design models improve users' attitude and motivate them as they increase alertness and concentration.

\subsection{Color and Light as Affective Factors}

Affective communication suggests a constantly updated exchange of emotionally charged information, where a human receives both verbal and nonverbal responses. The latter can be presented in the form of a set of environmental stimuli (at workplace, at home, etc.). As mentioned before, color and light should be mentioned as important stimuli in HCI. Our physical surroundings increase or reduce our stress, which in turn impacts our bodies in multiple ways. The following reports and monographs provide information about the evidences of emotional affects caused by environmental factors.

As it has been frequently reported in research, environmental factors include visual stimuli (e.g., color, light, video and animations), audio stimuli (e.g., noise, sound and music) and these environmental factors are graphically shown in Figure 4. Indeed, human emotions are influenced by many factors of different nature, not only by environment ones. But still this influence is constant and permanent. 


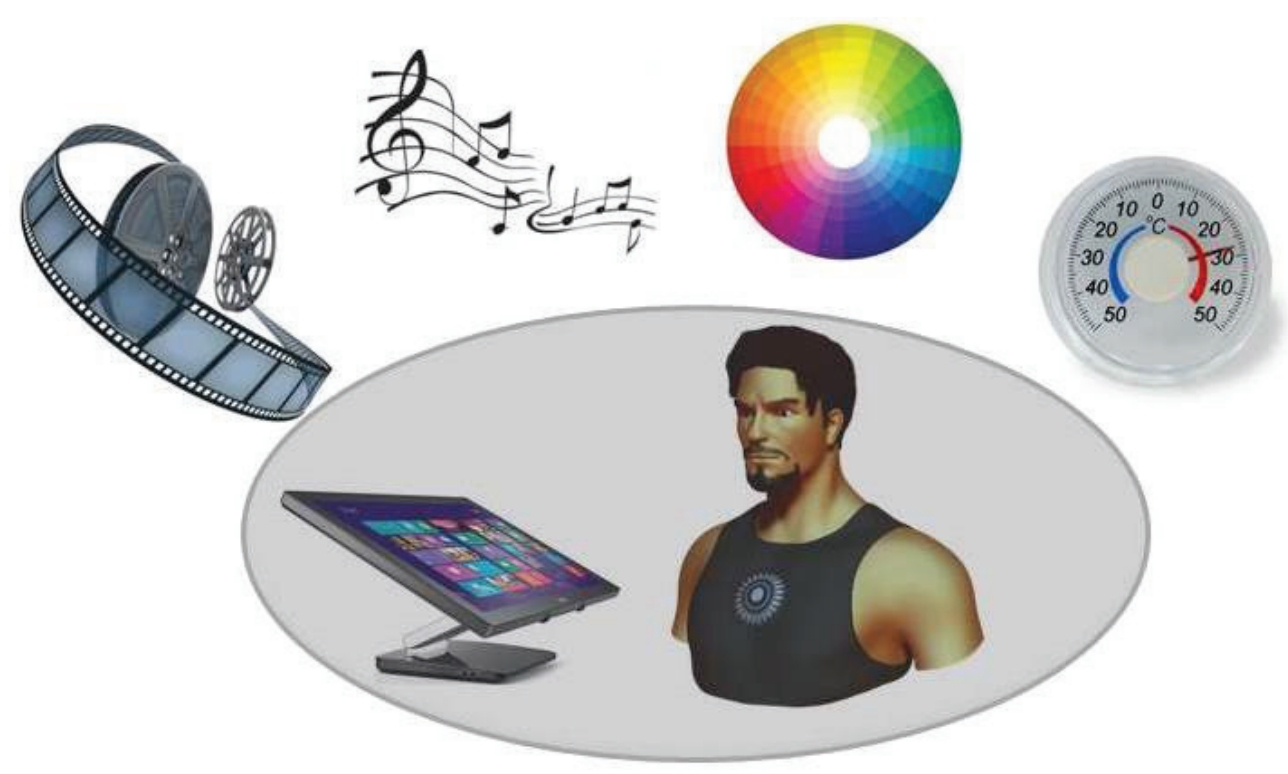

Figure 4. Environmental factors in affective computing.

Color and light perception affects our emotions and feelings, and this impact has been examined considerably with respect to color/mood associations [26,29,30], color meaning [24,47], color preference $[38,48]$, and color perception by particular demographic groups and psychologically healthy/unhealthy persons $[25,49,50]$.

\subsubsection{Influence of Light in Affective Computing}

Light is another main environmental factor that influences a user. Its affective impact has been widely researched and reported [51]. Exposure to bright light and luminance distribution contributes to affect our mood, and that is why it can be used to improve emotional states. Regulation of biochemical and hormonal rhythms are amongst the positive effects of the light on a human. For instance, light is used to calm seasonal affective disorders [52], vitamin D secretion, etc. The following findings inform about some further aspects of light perception. A significantly negative association between illuminance during daytime and Hamilton depression rating scale scores has been described [53]. On the contrary, no significant association between illumination in daytime and young mania rating scale has been found. The authors conclude that light can ameliorate low mood in healthy people.

Dumont and Beaulieu [54] have studied non-visual effects of light. They have carried out several experiments aimed at evaluating light exposure and illustrating how these concepts play a role in real-life situations. Their paper discusses the clinical relevance of light exposure in natural environments for mood, sleep and circadian disorders. Grandner et al. [55] reveals strong associations between exposure to light and quality of life as well as social and emotional functioning. Bauer et al. [51] discuss the time-course and durability of antidepressant effects of bright light in winter on depressive patients. Light exposures appear to cause higher clinical ratings of hypomanic symptoms in patients. Terman et al. [52] report about the results of light therapy combined with high-density negative air ionization. Both appear to act as specific antidepressants in patients with seasonal affective disorder. Meanwhile, 
Tuunainen et al. [56] evaluate the clinical effects of light therapy for non-seasonal depression. They confirm a moderate efficacy of light therapy, especially when administered in the morning.

\subsubsection{Influence of Color in Affective Computing}

Color is always present in our visual environment. Humans live consonant with their color choices that usually correspond to their internal rational and irrational feelings. Color preferences can be individual, collective and universal. Personal preferences correspond to the person's character as well as his/her moods and emotions. The evidences of color influence upon emotions have been discussed in a number of publications. Here we introduce some of the most relevant.

Gussakovsky et al. [57] show that plants are sensitive to the color of the light under which they are grown. The effect is maximal for red-enriched illumination. Elliot et al. [58] demonstrate that a brief exposure to red prior to a test unconsciously enhances the performance of the participants. Moreover, a link between red and avoidance motivation is discovered. The authors suggest that the red color influences behavior considerably; and care must be taken when using it in performance and achievement contexts. Some authors indicate antimicrobial and insecticide properties of blue color and light, reminding the tradition of painting houses and doors in blue in southern countries in order to get rid of insects [51]. In this context, it is worth mentioning an experiment which aimed to examine the possibility of enhancing antibody concentration and alleviate stress response in broilers. The experiment concludes in exposing broilers to white, red, green and blue light [59]. The results suggest that the combination of green and blue monochromatic light improves the broilers' immune function.

Table 1. Color in visual affect recognition.

\begin{tabular}{|c|c|c|}
\hline Reference & Emotion(s) & Color Description Model and Color Sample \\
\hline Ou et al. [60] & $\begin{array}{l}\text { Semantic scales warm/cool, heavy/light, } \\
\text { active/passive, and like/dislike }\end{array}$ & Two-color model, CIELAB; 190 color pairs \\
\hline Gao et al. [38] & 12 emotion variables (word pairs) & Munsell color system, SCOTDIC PLUS 2000 system; 214 color samples \\
\hline Hanada [61] & $\begin{array}{l}\text { Semantic word pairs "bright-dark" (activity } \\
\text { factor), "like-dislike" (evaluation factor) } \\
\text { and "strong-weak" (potency factor) }\end{array}$ & $\begin{array}{l}2 \text { datasets with different stimuli and scales; } 27 \text { color pairs, composed of } \\
\text { eight colors (red, blue, green, yellow, orange, cyan, magenta, and violet); } \\
\text { luminances and CIE xy color coordinates }\end{array}$ \\
\hline Lechner et al. [62] & $\begin{array}{l}\text { Attributes associated with emotional } \\
\text { responses }\end{array}$ & $\begin{array}{l}\text { Brightness level (light, medium, dark) and nine hues (red; orange; } \\
\text { yellow/orange; yellow; green; blue/green; blue; purple; and } \\
\text { white/gray/black) }\end{array}$ \\
\hline Choi et al. [63] & Heart rate variability (HRV) & Blue, red and white colored fluorescent lights \\
\hline Xin et al. [37] & $\begin{array}{l}\text { Color emotions described in } 12 \text { opponent } \\
\text { word pairs }\end{array}$ & SCOTDIC PLUS 2000 system, Munsell color system; 218 color samples \\
\hline Küller et al. [32] & 12 emotion variables (word pairs) & $\begin{array}{l}\text { Working environments with no color at all; with neutral colors; with not } \\
\text { very vivid color; very colorful and vivid }\end{array}$ \\
\hline Solli and Lenz [64] & $\begin{array}{l}\text { Three emotion factors: activity, weight, } \\
\text { and heat }\end{array}$ & RGB space; 567 samples \\
\hline Bonnardel et al. [41] & $\begin{array}{l}\text { Color emotion based on the analysis of } \\
\text { color preference questionnaires }\end{array}$ & $\begin{array}{l}\text { Self-created color space, where value and chroma are controlled, and } \\
\text { hue parameter are changed with a constant physical difference in hue of } \\
20 \text { grades, with addition of black and white, totally } 23 \text { home page color } \\
\text { schemes }\end{array}$ \\
\hline
\end{tabular}


Color's effect on emotional state can be measured indirectly by means of affective semantic words and word combinations. Table 1 contains references to many studies which are based on this approach. An analysis of this table reveals some common trends in the organization of experiments on color emotion. First, an indirect emotion assessment is widely used. It means that an emotion is evaluated with semantic words. They usually include, but are not limited to, the following pairs: "warm/cold", "heavy/light", "like/dislike", and "soft/hard".

Not many studies presented in Table 1 use physiological sensors for color-based emotion evaluation. For example, Choi et al. [63] receive information about heart rate variability to detect an emotion. Factor and correlation analysis (e.g., [32,38,60,61,64]) and ANOVA (e.g., [32,41,63]) head the list of the most widely used classification methods for color-based emotion regulation. RGB histograms for measuring statistical properties of color images are calculated in Solli and Lenz [64]. Lechner et al. [62] use decision tree models for classification. Firstly, results are calculated for countries based on brightness level choice. Next, they offer results for mean ranks of associations of hues given a brightness level choice. Besides ANOVA, Bonnardel et al. [41] also performs cluster analysis for website designers to evaluate color preference. With the purpose of studying color emotion, Hanada [61] use factor analysis, and, in the case of failure, independent component analysis with intrinsic statistical properties of data such as kurtosis.

Referring to the number of participants of the tests, some authors conduct experiments on an international level. This is the case of: Ou et al. [60], where 223 participants from 8 countries are interviewed; Gao et al. [38], where the sample includes people from 7 different region groups; and Xin et al. [37], where the sample counts with people from Japan, Thailand, and Hong Kong. The most numerous inquiries mentioned are carried out by Lechner et al. [62] and Küller et al. [32], which count with 2021 subjects from Brazil, Canada, China, France, Germany, India, Italy, Japan, South Korea, Spain, the United Kingdom and the United States, and with 998 persons aged 18 to 65 years from Argentina, Saudi Arabia, Sweden and the UK. Nevertheless, other references report about shorter groups of participants, such as the one which consisted of 36 undergraduate or graduate students [61], 92 adult volunteers [63], and 52 observers in the pilot and 35 in the main study [64].

Affective modulation by means of color may be performed in accordance with the metamodel shown in Figure 5. Here, color influence produces emotional changes, which are reflected in the human face. They can also alter some voice characteristics as well as causing bodily changes $[65,66]$. In case wearable sensors are used, physiological indicators of changes in emotional state (skin temperature, electrodermal activity, skin impedance, heart beat rate, etc.) can be registered as reported in numerous studies (e.g., [67-69]). However, a considerable number of findings indicate that the visual channel is dominant for affective stimuli perception as well as for emotional state "reading" through visually detectable indicators [67,70]. Thus, the combination of the many possible indicators of emotion expression and experience facilitate the identification of the affective status. Figure 5 suggests a possibility of a feedback with the aim of affective regulation by changing color and/or light. 


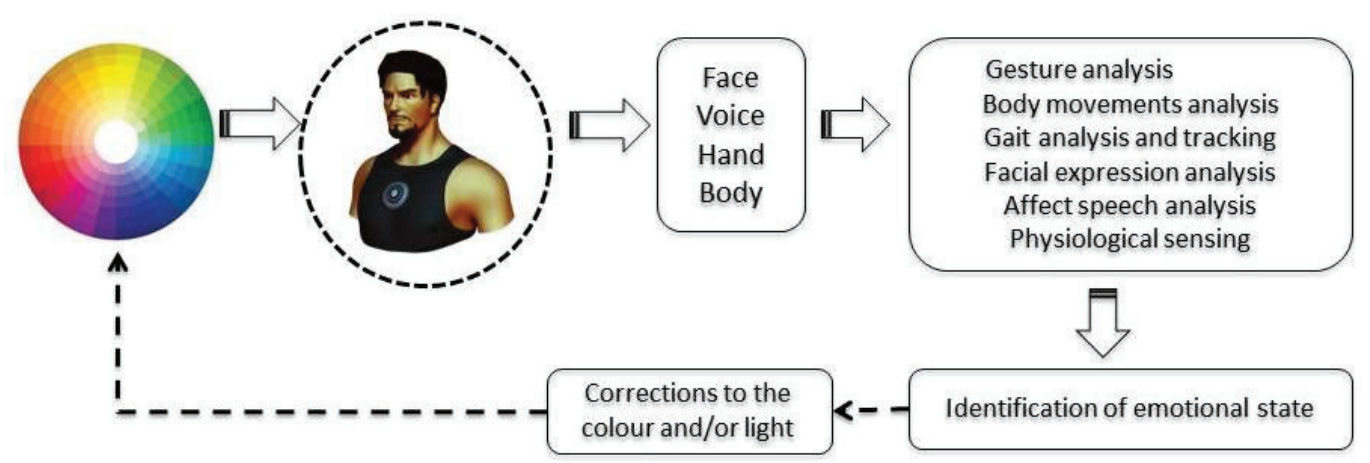

Figure 5. Metamodel for affective modulation through color.

It is important to note that some findings demonstrate that color features like chroma, hue or lightness cause an impact on emotions [25,37,71]. Indeed, it has been proved that the chromatic palette contains emotionally charged information as opposed to the achromatic one [25]. Lightness and chroma (though the degree of its influence is small in some cases) of color appear to dominate on most of the color emotion pairs in the research reported by Xin et al. [37]. Contrary to those two factors, the hue of a color does not affect the emotional state of the participants of the test. Similar results confirming the findings reported by Xin et al. [37] are obtained by Manav [48]. Ou et al. [60] point that older observers show strong preference to colors with higher chroma while younger people prefer achromatic colors.

Moreover, with respect to colors in particular, Manav [48] carries out a series of experiments and concludes that stimulating effects are made by pink and yellow color samples; green elicits the feelings of excitement, relaxation and vividness as well as of boredom and depression; blue is associated with calmness and coldness; red is stated as tiring and depressive, and together with violet they are named as striking colors. The author also finds a positive emotional response to black color for people aged 63 and over. [72] indicates that strong color (especially red) and patterns put the brain into a more excited state and may produce severe changes in the behavior of introverted people or in a bad mood. But, participants feel more positive in the red room than in the blue one, and also they felt more self control in a room painted in gray. In an experiment about color emotion with 98 participants [29], the green color attains the most positive emotions, followed by yellow and blue.

\subsection{Cultural and Demographic Aspects of Color and Light Perception}

\subsubsection{Universality of Cultural Aspects in Color and Light}

According to Darwin, gestures are culturally learned but some basic emotions are universal [8]. Ekman and Davidson [10], Ekman et al. [13] widened this theory after discovering universal emotions through conveying experiments with people from different cultures. On the other hand, Wierzbicka [11] argues that emotion analysis should depend on the explanation with very simple concepts that at least come closer both to being universal and to being semantic primitives. Her point is that much of the analysis of emotions conducted by psychologists has been in culture-dependent terms. As pointed out by Russell [73], emotions are categorized differently in different cultures and languages. However, it 
does not follow that emotion itself is not universal. Moreover, emotion categories are in fact extremely similar across cultures and languages [4].

Contrary to Ekman's basic emotions, Jack et al. [74] suggest that emotions are different. They performed their research with Western Caucasian and Eastern Asian participants. Indeed, the outcomes of the cluster analysis demonstrate clearly determined groups for Western Caucasians and dissimilarity and overlapping between categories for the Eastern Asian group. These conclusions are also confirmed with the results of the spatiotemporal location of emotional intensity and mutual information analysis. Indeed, the field of emotion theories is rich, but it has become common practice to accept the idea of Ekman's basic emotions for visual emotion recognition. Next, a non-exhaustive review that focuses on the theme of national and cultural aspects of color perception is offered.

A report focusing on cultural influence on color preference carried out by Sakamoto [75] presents the outcomes of the surveys performed in the Netherlands, Japan, Vietnam and China. Dutch participants prefer blue, green and red, while Japanese participants tend to like pink and pale green and blue. Japanese and Dutch have similar preferences to achromatic colors, especially to black for cell phones and clothes, and white and gray for computers. The color preferences of Chinese participants vary widely and no trend is detected.

Other important and interesting findings on the cultural aspects for color emotion are published in Ou et al. [60]. Eight observer groups, British, Taiwanese, French, German, Spanish, Swedish, Argentinean and Iranian, participate in an experiment to assess color emotion for two-color combinations using semantic scales warm/cool, heavy/light, active/passive, and like/dislike. The participants of the eight observer groups demonstrate similar responses for warm/cool, heavy/light, and active/passive word pairs, proving this way the hypothesis of universality of color emotion.

Gao et al. [38] investigate the differences and similarities between different groups from Hong Kong, Japan, Thailand, Taiwan, Italy, Spain and Sweden regarding color emotion. They evaluate emotions through 12 semantic word pairs. It is a peculiarity of this study on emotion that the authors assess the influence of color components as chroma, lightness and hue. As the previous study, this research confirms the existence of common trends of color emotion, but mentions several differences in responses. Thus, the authors point out the importance of lightness in the judgment of "heavy-light", and some peculiarities in judgments of other word pairs, influenced by chroma for Swedes, Italians, and Hong Kong subjects. The importance of the hue component is decisive for color temperature perception presented with the "warm-cool" variable.

To sum up, researchers who study emotional differences and color preferences for different regions and cultures come to similar conclusions. In general, there is a strong experimentally proved evidence of universality of color emotion regardless of cultural backgrounds. This is a very important statement, as it provides many opportunities for affective modulation. Thus, a cross-cultural tendency of color research and application in affective computing is crucial for developing emotionally intelligent machines and environments. In this way, particular color differences in color perceptions and preferences have been studied for people from different regions, including Western and Asian countries. Last but not least, cultural backgrounds affect though in a lesser extent emotions related to preferences "like/dislike" or altering more or less general trends. Nevertheless, these locally meaningful peculiarities of color emotion should be taken into account when special versions of affective computing modules are developed. 


\subsubsection{Universality of Demographic Aspects in Color and Light}

There are common trends in color perception shared by those who belong to a particular demographic group. Nevertheless, it is known that color preference changes with age. Colors preferred in infancy like yellow and red change into liking for blue or green $[75,76]$. Adults give more importance to the green color, which is related to happiness, or, in a lesser extent, to sadness [76].

Children react vividly to colors, which may explain their feelings [49]. In more detail, they show positive reactions to bright colors (e.g., pink, blue and red) and negative with dark colors (e.g., brown, black and gray). Positive emotional reactions of children to bright colors seem to progress and increase with age, especially for girls, whereas boys are more likely to have positive emotional associations with dark colors. Terwogt and Hoeksma [76] research color emotion associations in three age groups: 7-year-old, 11-year-old children, and adults. The preference in the youngest group includes blue, followed by red and yellow colors, which correlate with happiness. Black color is strongly associated with fear and angry. The second group demonstrates associations between feeling of happiness and blue and red color, as well as achromatic colors (black and white), equally associated with fear. Kurt and Osueke [77] study color preference of students aged between 17 and 24. They prefer living in an colorful setting where warm and cool colors should be present.

Two psychophysical experiments study how color emotion responses change with age [60]. The authors detect some points of difference for color preference with respect to chroma (the older like and the younger dislike colors with the high chroma). The greater difference in preference between the two groups is obtained for the emotional "like/dislike" scale, though the results for other semantic scales show similarities in emotional feelings. The authors hypothesize that the difference in color emotion response between the two age groups is limited, except for the achromatic colors, dark colors, and white. Indeed, these are more liked by younger observers than by elders.

\section{Conclusions}

This paper has introduced a review on the significance of light and color in HCI and affective computing. The importance of the study is related to the fact that light and color are omnipresent environmental factors which influence the human being.

The relation between $\mathrm{HCI} /$ affective computing and the emotions affected by light and color have been discussed in detail. Also, the color models generally used in affective computing have been briefly introduced. The review on the usage of color and light in affective computing has included detailed characteristics of the methods and recent research trends. The paper has ended up with a revision of papers related to the importance of light and color from demographic, gender and cultural aspects points of view.

The most outstanding results of the review are commented next. Firstly, color influences emotions irrationally or "mildly". However, as color is always present in our environments, its influence is constant, like in the cases of painted rooms or colored lighting. It is used in numerous and varied application fields. For example, in case of seasonal affective disorder therapy; for personalized learning systems; as a tool to improving the efficiency of HCI, and so on. On the contrary, light is an active 
factor in comparison to color, which could be labeled as a more proactive and energetic factor. The combination of both light and color is beneficial for the aims of affective computing.

Although there are common cross-national trends in color perception, the usage of some particular colors for people from specific cultures or regions should be taken with caution. The same precaution should be applied to different demographic groups. For example, children may react in a different way than adults under the exposure to certain colors and color combinations.

Lastly, affective computing could cause significant improvements in HCI, contributing to enhance the usability of interfaces. Indeed, computer acquired emotional intelligence, even in its basic form, facilitates an implicit interaction between humans and machines.

\section{Acknowledgments}

This work was partially supported by Spanish Ministerio de Economía y Competitividad/FEDER under TIN2013-47074-C2-1-R grant.

\section{Author Contributions}

Both authors have made substantial contributions in the definition of the research line, as well as in the preparation of the manuscript.

\section{Conflicts of Interest}

The authors declare that there is no conflict of interests regarding the publication of this article.

\section{References}

1. Fernández-Caballero, A.; Latorre, J.; Pastor, J.; Fernández-Sotos, A. Improvement of the elderly quality of life and care through smart emotion regulation. In Ambient Assisted Living and Daily Activities; Springer: New York, NY, USA, 2014; pp. 348-355.

2. Castillo, J.; Fernández-Caballero, A.; Castro-González, A.; Salichs, M.; López, M. A framework for recognizing and regulating emotions in the elderly. In Ambient Assisted Living and Daily Activities; Springer: New York, NY, USA, 2014; pp. 320-327.

3. Picard, R. Affective Computing; The MIT Press: Cambridge, MA, USA, 1997.

4. Strongman, K. The Psychology of Emotion: From Everyday Life to Theory, 5th ed.; John Wiley \& Sons: Hoboken, NJ, USA, 2003.

5. Gerrig, R.; Zimbardo, P. Psychology And Life; Allyn and Bacon: Boston, MA, USA, 2002.

6. Barlassina, L.; Newen, A. The role of bodily perception in emotion: In defense of an impure somatic theory. Philos. Phenomenol. Res. 2014, 89, 637-678.

7. Brave, S.; Nass, C. Emotion in human-computer interaction. In The Human-Computer Interaction Handbook; Jacko, J., Sears, A., Eds.; Erlbaum: Hillsday, NJ, USA, 2002; pp. 81-96.

8. Darwin, C. The Expression of the Emotions in Man and Animals; John Murray: London, UK, 1872.

9. Neese, R. Evolutionary explanations of emotions. Hum. Nat. 1990, 1, 261-289. 
10. Ekman, P.; Davidson, R. The Nature of Emotion; Oxford University Press: New York, NY, USA, 1994.

11. Wierzbicka, A. Talking about emotions: Semantics, culture, and cognition. Cogn. Emot. 1992, 6, 285-319.

12. Shweder, R. "You're not sick, you're just in love": Emotions as an interpretive system. In The Nature of Emotions; Ekman, P., Davidson, R., Eds.; Oxford University Press: Oxford, UK, 1994; pp. 32-44.

13. Ekman, P.; Friesen, W.; O’Sullivan, M.; Chan, A.; Diacoyanni-Tarlatzis, I.; Heider, K.; Tzavaras, A. Universals and cultural differences in the judgment of facial expressions of emotion. J. Personal. Soc. Psychol. 1987, 53, 712-717.

14. Damasio, A. Descartes' Error: Emotion, Reason, and the Human Brain; Gosset/Putnam Press: New York, NY, USA, 1994.

15. Reeves, B.; Nass, C. The Media Equation: How People Treat Computers, Television, and New Media Like Real People and Places; CSLI Publications: Stanford, CA, USA, 1996.

16. Picard, R. Affective computing: Challenges. Int. J. Hum.-Comput. Stud. 2003, 59, 55-64.

17. Broekens, J.; Brinkman, W. AffectButton: A method for reliable and valid affective self-report. Int. J. Hum.-Comput. Stud. 2013, 71, 641-667.

18. Chittaro, L.; Sioni, R. Affective computing vs. affective placebo: Study of a biofeedback-controlled game for relaxation training. Int. J. Hum.-Comput. Stud. 2014, 72, 663-673.

19. Wichmann, F.; Sharpe, L.; Gegenfurtner, K. The contributions of color to recognition memory for natural scenes. J. Exp. Psychol.: Learn., Memory Cogn. 2002, 28, 509-520.

20. Salovey, P.; Mayer, J. Emotional intelligence. Imagin. Cogn. Personal. 1989, 9, 185-211.

21. Mayer, J.; Geher, G. Emotional intelligence and the identification of emotion. Intelligence 1996, 22, 89-113.

22. Kiselev, A.; Hacker, B.; Wankerl, T.; Ohmoto, Y.; Abdikeev, N.; Nishida, T. Integrating the emotional intelligence into the virtual technical support engineer. In Modeling Machine Emotions for Realizing Intelligence; Nishida, T., Faucher, C., Eds.; Springer: New York, NY, USA, 2010; pp. 157-178.

23. Picard, R.; Vyzas, E.; Healey, J. Toward machine emotional intelligence: Analysis of affective physiological state. IEEE Trans. Pattern Anal. Mach. Intell. 2001, 23, 1175-1191.

24. O'Connor, Z. Colour psychology and colour therapy: Caveat emptor. Color Res. Appl. 2011, 36, 229-234.

25. Jue, J.; Kwon, S.M. Does colour say something about emotions? Laypersons' assessments of colour drawings. Arts Psychother. 2013, 40, 115-119.

26. Harleman, M. Colour emotion in full-scale rooms. In Proceedings of the AIC 2004 Color and Paints, Interim Meeting of the International Color Association, Porto Alegre, Brazil, 3-5 November 2004; pp. 223-226.

27. Plutchik, R.; Conte, H. Circumplex Models of Personality and Emotions; American Psychological Association: Washington, DC, USA, 1997.

28. Schauss, A. Tranquilizing effect of color reduces aggressive behavior and potential violence. J. Orthomol. Psychiatry 1979, 8, 218-221. 
29. Kaya, N.; Epps, H. Color-emotion associations: Past experience and personal preference. In Proceedings of the AIC 2004 Color and Paints, Interim Meeting of the International Color Association, Porto Alegre, Brazil, 3-5 November 2004; pp. 31-34.

30. Saito, Y.; Tada, H. Effects of color images on stress reduction: Using images as mood stimulants. Jpn. J. Nurs. Sci. 2007, 4, 13-20.

31. Lee, C.; Andrade, E.; Palmer, S. Interpersonal relationships and preferences for mood-congruency in aesthetic experiences. J. Consum. Res. 2013, 40, 382-391.

32. Küller, R.; Ballal, S.; Laike, T.; Mikellides, B.; Tonello, G. The impact of light and colour on psychological mood: A cross-cultural study of indoor work environments. Ergonomics 2006, 49, 1496-1507.

33. CIE. International Commission on Illumination. Available online: http://www.cie.co.at/ (accessed on 16 February 2015).

34. Zollinger, H. Color: A Multidisciplinary Approach; Verlag Helvetica Chimica Acta: Zürich, Switzerland, 1999.

35. MCS. Munsell Color. Available online: http://dba.med.sc.edu/price/irf/Adobe_tg/models/ munsell.html (accessed on 16 February 2015).

36. NCS. Natural Colour System. Available online: http://www.ncscolour.com/ (accessed on 16 February 2015).

37. Xin, J.; Cheng, K.; Taylor, G.; Sato, T.; Hansuebsai, A. Cross-regional comparison of colour emotions. Part I: Quantitative analysis. Color Res. Appl. 2004, 29, 451-457.

38. Gao, X.P.; Xin, J.; Sato, T.; Hansuebsai, A.; Scalzo, M.; Kajiwara, K.; Guan, S.S.; Valldeperas, J.; Lis, M.; Billger, M. Analysis of cross cultural color emotion. Color Res. Appl. 2007, 32, 223-229.

39. Beale, R.; Peter, C. The role of affect and emotion in HCI. In Affect and Emotion in Human-Computer Interaction; Peter, C., Beale, R., Eds.; Springer: New York, NY, USA, 2008; pp. $1-11$.

40. Cyr, D.; Head, M.; Larios, H. Colour appeal in website design within and across cultures: A multi-method evaluation. Int. J. Hum.-Comput. Stud. 2010, 68, 1-21.

41. Bonnardel, N.; Piolat, A.; Le Bigot, L. The impact of colour on Website appeal and users' cognitive processes. Displays 2011, 32, 69-80.

42. Schmidt, A. Implicit human computer interaction through context. Pers. Technol. 2000, 4, 191-199.

43. Harbich, S.; Hassenzahl, M. Beyond task completion in the workplace: Execute, engage, evolve, expand. In Affect and Emotion in Human-Computer Interaction; Peter, C., Beale, R., Eds.; Springer: New York, NY, USA, 2008; pp. 154-162.

44. Jokinen, J. Emotional user experience: Traits, events, and states. Int. J. Hum.-Comput. Stud. 2015, $76,67-77$.

45. Lim, Y.K.; Donaldson, J.; Jung, H.; Kunz, B.; Royer, D.; Ramalingam, S.; Thirumaran, S.; Stolterman, E. Emotional experience and interaction design. In Affect and Emotion in Human-Computer Interaction; Peter, C., Beale, R., Eds.; Springer: New York, NY, USA, 2008; pp. 116-291. 
46. Walker, S.; Prytherch, D. How is it for you? (A case for recognising user motivation in the design process). In Affect and Emotion in Human-Computer Interaction; Peter, C., Beale, R., Eds.; Springer: New York, NY, USA, 2008; pp. 130-141.

47. Braem, H. Die Macht der Farben; Signum: Seedorf, Germany, 2012.

48. Manav, B. Color-emotion associations and color preferences: A case study for residences. Color Res. Appl. 2007, 32, 144-150.

49. Boyatzis, C.; Varghese, R. Children's emotional associations with colors. J. Genet. Psychol. 1994, $155,77-85$.

50. Albert, N.; Burke, J.; Bena, J.; Morrison, S.; Forney, J.; Krajewski, S. Nurses' uniform color and feelings/emotions in school-aged children receiving health care. J. Pediatr. Nurs. 2013, 28, 141-149.

51. Bauer, M.; Kurtz, J.; Rubin, L.; Marcus, J. Mood and behavioral effects of four-week light treatment in winter depressives and controls. J. Psychiatr. Res. 1994, 28, 135-145.

52. Terman, M.; Terman, J.; Ross, D. A controlled trial of timed bright light and negative air ionization for treatment of winter depression. Arch. Gen. Psychiatry 1998, 55, 875-882.

53. Terao, T.; Hoaki, N. Light can ameliorate low mood in healthy people. Psychopharmacology 2011, 213, 831, doi:10.1007/s00213-010-2046-x.

54. Dumont, M.; Beaulieu, C. Light exposure in the natural environment: Relevance to mood and sleep disorders. Sleep Med. 2007, 8, 557-565.

55. Grandner, M.; Kripke, D.; Langer, R. Light exposure is related to social and emotional functioning and to quality of life in older women. Psychiatry Res. 2006, 143, 35-42.

56. Tuunainen, A.; Kripke, D.; Endo, T. Light therapy for non-seasonal depression. Cochrane Database Syst. Rev. 2004, 2, CD004050.

57. Gussakovsky, E.; Shahak, Y.; Schroeder, D. Color of illumination during growth affects LHCII chiral macroaggregates in pea plant leaves. J. Photochem. Photobiol. 2007, 86, 121-130.

58. Elliot, A.; Maier, M.; Moller, A.; Friedman, R.; Meinhardt, J. Color and psychological functioning: The effect of red on performance attainment. J. Exp. Psychol.: Gen. 2007, 136, 154-168.

59. Zhang, Z.; Cao, J.; Wang, Z.; Dong, Y.; Chen, Y. Effect of a combination of green and blue monochromatic light on broiler immune response. J. Photochem. Photobiol. 2014, 138, 118-123.

60. Ou, L.C.; Luo, M.; Sun, P.L.; Hu, N.C.; Chen, H.S. Age effects on colour emotion, preference, and harmony. Color Res. Appl. 2012, 37, 92-105.

61. Hanada, M. Analyses of color emotion for color pairs with independent component analysis and factor analysis. Color Res. Appl. 2013, 38, 297-308.

62. Lechner, A.; Simonoff, J.; Harrington, L. Color-emotion associations in the pharmaceutical industry: Understanding universal and local themes. Color Res. Appl. 2012, 37, 59-71.

63. Choi, C.; Kim, K.; Kim, C.; Kim, S.; Choi, W. Reactivity of heart rate variability after exposure to colored lights in healthy adults with symptoms of anxiety and depression. Int. J. Psychophysiol. 2011, 79, 83-88.

64. Solli, M.; Lenz, R. Color emotions for multi-colored images. Color Res. Appl. 2011, 36, $210-221$.

65. Fernandez, R. A Computational Model for the Automatic Recognition of Affect in Speech. Ph.D. Thesis, Massachusetts Institute of Technology, Cambridge, MA, USA, February 2004. 
66. Karg, M.; Samadani, A.; Gorbet, R.; Kuhnlenz, K.; Hoey, J.; Kulic, D. Body movements for affective expression: A survey of automatic recognition and generation. IEEE Trans. Affect. Comput. 2013, 4, 341-359.

67. Zeng, Z.; Pantic, M.; Roisman, G.; Huang, T. A survey of affect recognition methods: Audio, visual, and spontaneous expressions. IEEE Trans. Pattern Anal. Mach. Intell. 2009, 31, 39-58.

68. Picard, R. Emotion research by the people, for the people. Emot. Rev. 2010, 2, 250-254.

69. Kukolja, D.; Popovic, S.; Horvat, M.; Kovac, B.; Cosic, K. Comparative analysis of emotion estimation methods based on physiological measurements for real-time applications. Int. J. Hum.-Comput. Stud. 2014, 72, 717-727.

70. Ambady, N.; Rosenthal, R. Thin slices of expressive behavior as predictors of interpersonal consequences: A meta-analysis. Psychol. Bull. 1992, 111, 256-274.

71. Xin, J.; Cheng, K.; Taylor, G.; Sato, T.; Hansuebsai, A. Cross-regional comparison of colour emotions. Part II: Qualitative analysis. Color Res. Appl. 2004, 29, 458-466.

72. Küller, R.; Mikellides, B.; Janssens, J. Color, arousal, and performance-A comparison of three experiments. Color Res. Appl. 2009, 34, 141-152.

73. Russell, J. Core affect and the psychological construction of emotion. Psychol. Rev. 2003, 110, 145-172.

74. Jack, R.; Garrod, O.; Yu, H.; Caldara, R.; Schyns, P. Facial expressions of emotion are not culturally universal. Proc. Natl. Acad. Sci. USA 2012, 109, 7241-7244.

75. Sakamoto, K. Cultural influence to the color preference according to product category. In Proceedings of the International Conference on Kansei Engineering and Emotion Research, Linköping, Sweden, 11-13 June 2014; pp. 1427-1434.

76. Terwogt, M.; Hoeksma, J. Colors and emotions: Preferences and combinations. J. Gen. Psychol. 1995, 122, 5-17.

77. Kurt, S.; Osueke, K. The effects of color on the moods of college students. SAGE Open 2014, 4, doi:10.1177/2158244014525423.

(c) 2015 by the authors; licensee MDPI, Basel, Switzerland. This article is an open access article distributed under the terms and conditions of the Creative Commons Attribution license (http://creativecommons.org/licenses/by/4.0/). 